\title{
Isomerien in der Cinchoningruppe von
}

\author{
Zd. H. Skraup, \\ w. M. k. Akad.
}

Aus dem chemischen Institute der Universität Graz.

(Vorgelegt in der Sitzung am 12. Mai 1899.)

Im Laufe der letzten Jahre sind aus dem Cinchonin nicht weniger wie $15 \mathrm{mit}$ ihm isomere Alkaloide dadurch dargestellt worden, dass es mit starken Säuren erhitzt oder mit Halogenwasserstoff additionell verbunden und dieser dann wieder abgespalten worden ist.

Sollten diese Basen, wie aus verschiedenen Gründen wahrscheinlich ist, optische Stereoisomere des Cinchonins sein, so ist die Existenz von 16 isomeren Formen mit der Constitution des Cinchonins unvereinbar, wie sie gegenwärtig anzunehmen ist. Nach dieser sind 3 asymmetrische Kohlenstoffatome vorhanden, demnach 8 optische und 4 racemische Isomere möglich. Nimmt man weiter an, dass einige dieser Basen structurell mit dem Cinchonicin (Cinchotoxin) identisch, also Ketoformen mit bloss 2 assymmetrischen Kohlenstoffatomen wären, so kämen noch 4 weitere optisch active und 2 racemische Formen hinzu. Im Ganzen wären dann 12 active und 6 racemische Formen möglich, während mit dem Cinchonicin, das wahrscheinlich identisch mit dem Cinchotoxin von v. Miller und Rhode ist, 17 isomere und durchwegs optisch active Formen beschrieben worden sind. ${ }^{1}$ Dabei ist keine

1 o. Hesse nimmt (Liebig's Ann., 276,89) an, dass wenn im Cinchonin, Cinchonidin, Chinin und Chinidin je 2 assymmetrische Kohlenstoffatome vorhanden sind, für jedes der genannten Alkaloide 16 isomere Formen existiren können, was mir mit unseren Vorstellungen über optische. Isomerie unvereinbar erscheint. Dasselbe nehmen E. Jungfleisch und Léger an (Les. Alcaloïdes. des Quinquinas, p. 242). 
Rücksicht darauf genommen, dass möglicherweise auch das Cinchonidin und einzelne aus diesem durch Umlagerung dargestellte Basen auch stereoisomere Formen des Cinchonins sein könnten.

Gelegentlich anderer Untersuchungen haben sich Anhaltspunkte dafür ergeben, dass einige der beschriebenen Isomeren keine chemischen Individuen sind, sondern zusammenfallen, und dies hat Veranlassung geboten, auch die übrigen auf ihre Existenzberechtigung zu überprüfen.

Von den 15 früher erwähnten Basen sind wie schon aus den veröffentlichten Eigenschaften hervorgeht, das $\alpha$ - und $\beta$-Isocinchonin von Hesse (Cinchonilin und Cinchonigin von Jungfleisch und Léger), das $\delta$-Cinchonin von Jungfleisch und Léger, das $\varepsilon$-Cinchonin und Tautocinchonin von v. Cord i e r von einander und vom Cinchonin bestimmt verschieden und als selbständige Individuen aufrechtzuhalten. Das Apocinchonin, sowie das Apoiso- und das Isoapocinchonin von Hesse fallen aber mit dem Allocinchonin (früher Pseudocinchonin) von Lippmann und Fleissner zusammen, welches das sechste, mit dem Cinchonin das siebente Isomere ist.

Das Pseudocinchonin von Hesse ist, wie Herr v. Arlt festgestellt hat, ${ }^{1}$ überhaupt kein Isomeres des Cinchonins, sondern nichts anderes als Cinchotin (Hydrocinchonin von Hesse), welches bekanntlich dem käuflichen Cinchonin stets beigemischt ist. Wahrscheinlich gilt dies auch vom Cinchonifin von Jungfleisch und Lěger.

Das Diapocinchonin von Hesse ist, wie es schon Jungfleisch und Léger behauptet ${ }^{2}$ haben, Hesse ${ }^{3}$ später aber wieder bestritten hat, ein Gemisch verschiedener Basen, in welchen $\alpha$ - und $\beta$-Isocinchonin mit Bestimmtheit nachgewiesen werden konnten. Das Homocinchonin von Hesse ist im Wesentlichen Cinchonin, welchem es in Wirklichkeit noch viel ähnlicher ist, als schon aus den Angaben von Hesse hervorgeht.

1 Monatshefte für Chemie, 1899.

2 Compt. rend., 114, 1192.

3 Liebig's Ann., 276, 118. 
Das $\beta$ - und $\gamma$-Cinchonin von $\mathrm{Pum}^{1}$ endlich habe ich bei Wiederholung seiner Versuche nicht erhalten, sondern dafür Allocinchonin. Vermuthlich sind die von Pum beschriebenen Basen Gemische von Cinchonin und einer zweiten, bisher nicht beschriebenen Base gewesen, welche neben Allocinchonin entsteht. Diese ist ein neues Isomeres des Cinchonins und soll bei einer späteren Gelegenheit beschrieben werden.

Über das Dicinchonin, welches Hesse ${ }^{2}$ aus den Mutterlaugen von der Fabrication des Chininsulfates abgeschieden hat, fehlen mir Erfarungen. Nach der Beschreibung von Hes se könnte es $\alpha$-Isocinchonin sein; ist es aber ein selbständiges Individuum, dann liegt noch immer nicht der geringste Anhaitspunkt vor, dass es gerade zum Cinchonin in irgend welcher näheren Bestimmung steht.

\section{$\alpha$ - und $\beta$-Isocinchonin.}

$\mathrm{Zu}$ den bekannten auffallenden Unterschieden dieser zwei im Gegensatz zum Cinchonin in Äther sehr leicht löslichen Basen sei noch der zugefügt, dass das Bisulfat des $x$-Isocinchonins in Wasser und Alkohol spielend leicht löslich ist und nicht krystallisirt, das des $\beta$-Isocinchonins dagegen, nach der Formel $\mathrm{C}_{19} \mathrm{H}_{22} \mathrm{~N}_{2} \mathrm{O} . \mathrm{H}_{2} \mathrm{SO}_{4}+4^{1} /{ }_{2} \mathrm{Aq}$ zusammengesetzt, in Wasser zwarauch ziemlich leicht, in Alkohol aber fast unlöslich ist und in zugespitzten weissen Prismen sehr leicht krystallisirt.

Beide Basen krystallisiren aus trockenem alkoholfreien Äther sehr leicht, die $\alpha$-Verbindung in grossen, dicken, flächenreichen Tafeln, die $\beta$ - in langgestreckten Prismen. Aus wasseroder alkoholhältigem Äther bleiben beide in Form schwer krystallisirender Syrupe zurück.

Man unterscheidet beide am sichersten an derverschiedenen Löslichkeit ihrer sauren Jodhydrate, von denen das der $\alpha$-Base in kaltem Wasser sehr schwer, das der $\alpha$-Verbindung sehr leicht löslich ist. Auch die Trennung beider Basen geht in Form der Jodhydrate sehr leicht, auf welche Verhältnisse schon von $\mathrm{H}$ ess e aufmerksam gemacht worden ist.

1 Monatshefte für Chemie, 1892, 683 und 689.

Liebig's Ann. 276, 119. 
Das $\delta$-Cinchonin, welches von Jung fleisch und Lég er, ${ }^{1}$ sowie später von v. Cordier ${ }^{2}$ durch Zerlegung des Hydrobromcinchonins gewonnen, und von v. Arlt ${ }^{3}$ unter den Producten der Einwirkung von Salzsäure auf Cinchonin aufgefunden worden ist, sowie das $\varepsilon$-Cinchonin, das v. Cordier ebenfalls aus Hydrobromcinchonin gewonnen hat, haben untereinander und mit dem $\beta$-Isocinchonin äusserlich so viel Ähnlichkeit, dass Verwechslungen nicht unmöglich sind. Sie sind aber alle drei durch sehr verschiedenes Drehungsvermögen charakterisirt, und das $\delta$ - und $\varepsilon$-Cinchonin sind in Äther viel schwieriger löslich als $\beta$-Cinchonin und schmelzen viel höher wie dieses. $\delta$ - und $\varepsilon$-Cinchonin sind sicher nur durch ihr sehr verschiedenes optisches Verhalten auseinanderzuhalten.

In folgender. Tabelle sind die Daten gesammelt, welche zur Erkennung der vier Basen am wichtigsten sind:

\begin{tabular}{|c|c|c|c|c|c|c|}
\hline & & & & In Wass & $r$ ist das & \\
\hline & F.P. & Alkohol & $\frac{\text { keit in }}{\text { Äther }}$ & $\begin{array}{l}\text { Chlorhydrat } \\
\text { (Base }+\mathrm{HCl} \text { ) }\end{array}$ & $\begin{array}{l}\text { Jodhydrat } \\
\text { (Base+2HJ) }\end{array}$ & $\begin{array}{l}\text { in Alkohol } \\
\text { für } p=3 \text {, } \\
\quad t=20\end{array}$ \\
\hline -Isocinchonin & 127 & sehr leicht & leicht & leicht löslich & $\begin{array}{l}\text { sehr schwer } \\
\text { 1öslich }\end{array}$ & $+53^{\circ}$ \\
\hline Isocinchonin & 127 & . & , & schwierig & $\begin{array}{l}\text { sehr leicht } \\
\text { löslich }\end{array}$ & $-60^{\circ}$ \\
\hline Cinchonin. & 151 & ${ }^{\prime}$ & schwierig & schwer löslich & $一$ & $+125^{\circ}$ \\
\hline Cinchonin. & 150 & $\gg$ & $\gg$ & > & - & $+58^{\circ}$ \\
\hline
\end{tabular}

Das Tautocinchonin von v. Cordier ${ }^{4}$ hat mit dem Cinchonin grosse Ähnlichkeit. Krystallwassergehalt, Form und Löslichkeit der Saize, Schmelzpunkt und optisches Drehungsvermögen sind vom Cinchonin beziehlich dessen Salze so wenig unterschieden, dass es als Cinchonin aufgefasst werden müsste, wenn nicht in der Löslichkeit der freien Basen beziehlich des Sulfides constante Unterschiede bestünden. v. Cordier hat unter verschiedenen Bedingungen übereinstimmend gefunden, dass das Sulfat bei $20^{\circ}$ in Wasser leichter löslich ist,

\footnotetext{
1 Compt. rend., 118, 29.

2 Monatshefte für Chemie, 1898, 472.

3 Monatshefte für Chemie, 1899.

4 Monatshefte für Chemie, 1898, 465.
} 
(1:51) als Cinchoninsulfat $(1: 75)$, die Basen bei $20^{\circ}$ in absolutem Alkohol dagegen wesentlich schwerer $(1: 142)$ wie reines Cinchonin $(1: 115)$.

Die grössere Löslichkeit des Sulfates allein betrachtet, liesse sich durch die Annahme erklären, dass nicht völlig reines Cinchoninsalz vorliegt, dem steht aber die Thatsache entgegen, dass die freie Base schwieriger löslich ist als Cinchonin. Und hiezu kommt noch, dass verschiedene Fractionirungen, so durch Umwandlungen in das Chlorhydrat oder Jodhydrat, an der Schwerlöslichkeit des Tautocinchonins nichts geändert haben.

Immerhin ist die Ähnlichkeit von Cinchonin und Tautocinchonin sehr auffallend.

\section{Allocinchonin identisch mit Apo-, Apoiso- und Isoapo- cinchonin.}

Das Allocinchonin von Lippmann und Fleissner ${ }^{1}$ ist insbesondere dadurch charakterisirt, dass es ein in Wasser auffallend schwer lösliches (bei $20^{\circ}$ etwa $1: 120$ ) neutrales Sulfat $\left(\mathrm{C}_{19} \mathrm{H}_{22} \mathrm{~N}_{2} \mathrm{O}\right)_{2} \mathrm{H}_{2} \mathrm{SO}_{4}$ liefert, das in weissen Nadeln krystallisirt und ferner, dass die Base bei $216^{\circ}$ schmilzt. Auf die Ähnlichkeit dieser Base mit dem Apoisocinchonin ${ }^{2}$ von $\mathrm{Hesse}$ haben vor einiger Zeit schon W. Königs und M. Hoppner (Ber. 31, 2361) aufmerksam gemacht.

Es hat sich nun herausgestellt, dass Allocinchonin, genau nach den Angaben von Lippmann und Fleissner dargestellt, bei $p=3$ und $t=20^{\circ}$ in absolutem Alkohol nahezu dasselbe Drehungsvermögen, d. i. $\alpha_{D}=+164 \cdot 8^{\circ}$ hat, wie das Apoisocinchonin, für welches Hesse unter den erwähnten Bedingungen $\alpha_{D}$ mit $166 \cdot 8$ angibt.

Weiter zeigte sich, dass Apoisocinchonin, nach den Angaben von Hesse aus $\beta$-Isocinchonin dargestellt, ein Sulfat liefert, das, wiederholt aus Wasser umkrystallisirt, durchaus nicht, wie Hesse angibt, in Wasser »ziemlich leicht» löslich ist, da es bei $20^{\circ} 104$ Theile Wasser zur Lösung bedurfte. In Folge

\footnotetext{
1 Monatshefte für Chemie, 1893, 371 und Berl. Ber., 1893, 2005.

2 Liebig's Ann., 276, 99.
} 
dessen liegt nicht der geringste Grund vor, die beiden Basen als verschieden anzusehen.

Aber auch das Isoapocinchonin von $\mathrm{Hesse}^{1}$ ist im Wesentlichen Allocinchonin. Es wurde nach den Angaben von O. Hesse durch Erhitzen von $13 g^{\circ}$ saurem salzsauren Hydrochlorcinchonin mit $220 \mathrm{~g}$ Wasser durch 6 Stunden auf 140 bis $150^{\circ}$ dargestellt. Von der mit Natriumhydroxyd ausgefällten Base waren nur $4.5 \mathrm{~g}$ in Äther unlöslich; die ätherlösliche Hauptmenge enthielt erhebliche Mengen von $\alpha$-Cinchonin, die in Form des sauren Jodhydrates abgeschieden wurden.

Die in Äther unlösliche Base wurde durch Kochen mit weingeistigem Kali von einer chlorhaltigen Beimengung befreit und dann wiederholt aus Alkohol umkrystallisirt. Der Schmelzpunkt war anfänglich $222^{\circ}$, stieg dann allmälig auf 231 , also ungefähr bis dahin, wo Hesse den Schmelzpunkt angibt; da aber war die Menge auf $0.15 \mathrm{~g}$ zusammengeschmolzen und die Hauptmenge in den alkoholischen Mutterlaugen enthalten. Diese, mit verdünnter Schwefelsäure neutralisirt, gaben in reichlicher Menge das charakteristische Sulfat des Allocinchonins, denn nach wiederholtem Umkrystallisiren aus Wasser brauchte es bei $20^{\circ} 112$ Theile Wasser zur Lösung, und die verschiedenen Fractionen schieden Basen ab, die, aus Alkohol krystallisirt, innerhalb der Grenzen $213-217^{\circ}$, meist bei $215^{\circ}$ schmolzen.

Und ganz Ähnliches zeigte sich beim Apocinchonin. ${ }^{2}$

Dieses ist von Hesse beim Erhitzen von Cinchonin mit Salzsäure vom specifischen Gewichte $1 \cdot 125$ auf $140-150^{\circ}$ neben Apoisocinchonin, d. i. Allocinchonin, erhalten worden; sein Sehmelzpunkt ist mit $228^{\circ}$ angegeben. Bei Wiederholung des Versuches (mit $30 \mathrm{~g}$ Cinchonin) fand sich, dass nicht unerheblich Hydrochlorcinchonin entsteht, von welchem $3 g$ des sauren salzsauren Salzes im erkalteten Rohr auskrystallisirt waren. Das Filtrat, so wie es Hesse angibt, mit Alkohol vermischt und mit Ammoniak gefällt, gab $10 \mathrm{~g}$ einer. Fällung, die, weil stark chlorhältig, mit alkoholischem Ätzkali gekocht wurde: Nach dem Erkalten fielen nur mehr $7 \mathrm{~g}$ vom Schmelzpunkte

\footnotetext{
1 Liebig's Ann., 276, 116.

2 Ebenda 276, 115 und 205, 330 .
} 
$221^{\circ}$ aus, der nach öfterem Umkrystallisiren auf $242^{\circ}$ anstieg, wobei aber Alles bis auf einen geringen Rest in den Mutterlaugen geblieben war, und diese, mit Schwefelsäure neutralisirt. gaben neben nicht unbeträchtlichen Mengen eines nicht krystallisirenden, in Wasser spielend löslichen Sulfates wieder die charakteristischen Fäden des Allocinchoninsulfates, und diese verschiedenen Krystallisationen schieden Basen ab, deren Schmelzpunkt $z$ wischen $213-218^{\circ}$ lag. Das Apocinchonin ist also im Wesentlichen wieder Allocinchonin. Für die vier als identisch erkannten Basen muss eine Bezeichnung gewählt werden; der Name Allocinchonin empfiehlt sich am besten, weil Lippmann und Fleissner die Base rein in den Händen hatten, und weil die anderen Namen, wie Isoapocinchonin, unnöthig schleppend sind.

Die Eigenschaften der Base, so ihr Schmelzpunkt, ihre Löslichkeit in Alkohol, die Löslichkeit des Sulfates in Wasser, scheinen von geringfügigen Verunreinigungen stark beeinflusst zu werden, die schwierig zu entfernen sind, und dies erklärt, warum die Eigenschaften manchmal recht erheblich abweicheri: So habe ich bei genauester Beobachtung der Vorschrift von Hes se für die Darstellung des »Apoisocinchonins « dessen Schmelzpunkt constant bei $209-210^{\circ}$, und nicht, wie Hesse bei seinem ganz gleich hergestellten Präparat und ich auch sonst meistens, bei $216^{\circ}$ gefunden.

Was den Krystallwassergehalt des Sulfates anbelangt, sei erwähnt, dass aller Wahrscheinlichkeit nach das Salz Krystallwasser besitzt, aber so leicht verliert, dass schon nach einigem Liegen an der Luft, ja nach in der gewöhnlichen Art erfolgtem sorgfältigen Abpressen $z$ wischen Filtrirpapier, dieses ganz oder grösstentheils abgegeben ist. So fand ich im "Isoapocinchoninsulfat " bloss $1 \cdot 67$ Wasser, während $\mathrm{Hesse}$ die Formel $+2 \mathrm{H}_{2} \mathrm{O}$, $4.61 \%$ gefunden hat, im Sulfat des "Apoisocinchonins", welches denselben Krystallwassergehalt haben soll, $1.04 \%$, dafür im "Allocinchoninsulfat « nach Lip pmann und Fleissner, welches Krystallwasser frei beschrieben ist, nach sehr rascheni Abpressen 7.93, nach nochmaligem Abpressen 2.09\% und nach einem drittmaligen $0.2 \%$ Wasser. 


\section{Pseudocinchonin und Cinchonifin.}

Herr v. Arlt hat festgestelit, dass die von Hesse unter dem Namen Pseudocinchonin beschriebene Base im Wesentlichen Cinchotin (Hydrocinchonin) ist, welchem Allocinchonin, sowie andere Basen anhaften. Wahrscheinlich ist auch das Cinchonifin von Jungfleisch und Léger ${ }^{1}$ nichts anderes als Cinchotin. Die sehr dürftigen Angaben, die über das Cinchonifin vorliegen, lassen keinen Unterschied zwischen dem Cinchotin und Cinchonifin erkennen; so schmilzt ersteres bei $277^{\circ}$, letzteres bei $274^{\circ}$, beidemale corrigirt, und auch die angegebenen Löslichkeitsverhältnisse sind sehr ähnlich. Dafür spricht weiter, dass, wenn man vom öfters umkrystallisirten Cinchoninbisulfat, welches Cinchotin nicht oder doch nur in sehr geringer Menge enthalten kann, ausgeht und dieses so, wie es Jungfleisch und Léger angeben, mit mässig verdünnter Schwefelsäure anhaltend kocht, eine Base von den Eigenschaften des Cinchonifins nur in äusserst geringen Mengen entsteht. Aus $150 \mathrm{~g}$ Cinchoninbisulfat, respective aus dem Jodhydrat, welches nach dem am citirten Orte mitgetheilten Verfahren im Wesentlichen Cinchonifin enthalten soll, liessen sich allerdings $11 \mathrm{~g}$ Base abscheiden, deren Schmelzpunkt aber unscharf bei $230^{\circ}$ lag und auf etwa $258^{\circ}$ erst dann gestiegen war, als die Menge durch öfteres Umkrystallisiren aus Alkohol auf 0.35 reducirt war. Die verschiedenen alkoholischen Mutterlaugen, mit verdünnter Schwefelsäure neutralisirt, gaben eingedampft erhebliche Mengen der charakteristischen weichen Krystallfäden vom Sulfat des Allocinchonins, aus welchem die abgeschiedenen Basen den Schmelzpunkt 213-214 oder einen wenig abweichenden hatten.

Die in Form des Jodhydrates isolirte Base ist also im Wesentlichen wieder Allocinchonin. Die leichter schmelzenden alkoholschwerlöslichen Fractionen waren in so geringer Menge vorhanden, dass eine nähere Charakterisirung unmöglich war. Die Vermuthung, dass das Cinchonifin nichts anderes wie Cinchonin ist, gewinnt an Wahrscheinlichkeit dadurch, dass 
Jungfleisch und Léger von nicht besonders gereinigtem, daher cinchotinhältigem Cinchonin ausgegangen sind. Cinchotin ist gegen Schwefelsäure widerstandsfähiger als Cinchonin, es ist in Äther und verdünntem Weingeist praktisch unlöslich, es muss also in jene Fractionen übergehen, welche die genannten Chemiker als ein Gemisch von Cinchonifin und dem Hesseschen Apocinchonin, aufgefasst haben, welches, wie früher nachgewiesen worden ist, im Wesentlichen Allocinchonin ist.

Wenn nun möglichst cinchotinfreies Cinchonin fast ausschliesslich Allocinchonin, ein mit Cinchotin vermischtes, neben Allocinchonin eine in Alkohol sehr schwer lösliche und sonst dem Cinchotin sehr ähnliche Base liefert, ist der Verdacht, diese sei nichts anderes als Cinchotin, vollauf gerechtfertigt, und die Individualität des Cinchonifins daher durch besondere Versuche erst zu rechtfertigen.

\section{Homocinchonin.}

Diese Base entsteht nach Hesse ${ }^{1}$ beim Erhitzen von Cinchonin mit wässeriger Schwefelsäure von $25 \%$ auf $1.40^{\circ}$ durch 6 Stunden. Nach der Beschreibung von Hesse ist es dem Cinchonin äusserst ähnlich; so ist der Krystallwassergehalt der Salze ganz so wie beim Cinchonin, ihr Habitus kaum verschieden, ebenso Drehungsvermögen, Schmelzpunkt u. s. w.

Das nach der Vorschrift von Hesse dargestellte Sulfat bildet gleich dem Cinchoninsulfat derbe, doch längere und melor verzerrte Prismen, die auch in der Löslichkeit in Wasser etwas differiren. Während bei $20^{\circ}$ Cinchoninsulfat 75 Theile braucht, löst sich Homocinchoninsulfat schon in 67-68 Theilen, und dieses Löslichkeitsverhältniss findet man merkwürdigerweise nicht nur bei verschiedenen Darstellungen, sondern auch bei verschiedenen, durch systematisches Umkrystallisiren erhaltenen Fractionen; so war es bei drei aufeinanderfolgenden Anschüssen $1: 67: 69: 68$.

Dieser Unterschied vom Cinchonin wird geringer, wenn man die Darstellung des Homocinchonins nur etwas abändert,

1 Liebig's Ann., 276, 103. 
so unter Beibehaltung aller anderen Umstände statt 25 percentiger nur 10 percentige Schwefelsäure nimmt. In diesem Falle erhält man ein Sulfat, das dem des Cinchonins schon äusserlich und ausserdem darin viel mehr ähnelt, dass es sich in 72 Theilen Wasser löst.

Hesse hat mitgetheilt, dass das saure Chloroplatinat des Homocinchonins amorph ist und sich dadurch von dem des Cinchonins unterscheidet. Diesen Unterschied konnte ich nicht beobachten. Beide Alkaloide geben, in gleicher Weise mit Platinchlorid gefällt, körnige, unter dem Mikroskop gleich aussehende Niederschläge, die, in heisser verdünnter Salzsäure gelöst, beim Erkalten in orangegelben Schuppen ausfielen, die unter dem Mikroskop gleichgeformte, schmale, viereckige Platten zeigten.

Die Differenzen zwischen Cinchonin und Homocinchonin sind demnach nicht grösser, als sie allgemein zwischen chemisch reiner und etwas verunreinigter Substanz bestehen, und liegt daher kein Grund vor, das Homocinchonin als Individuum länger zu führen.

Es sei noch bemerkt, dass sowohl beim Erhitzen mit 25 percentiger, als auch mit 10 procentiger Schwefelsäure $\alpha$-Isocinchonin entsteht, wie schon Hesse erwähnt hat, daneben aber auch in kleiner Menge ein Körper von den Eigenschaften der Sulfonsäure, wie sie beim Lösen von Cinchonin in concentrirter Schwefelsäure sich bildet, und beide Substanzen in Mengen, welche der Concentration der beim Erhitzen verwendeten Schwefelsäure proportional sind.

\section{Diapocinchonin.}

Dieses entsteht nach $\mathrm{H}$ ess e ${ }^{1}$ beim Erhitzen von Cinchonin oder $\alpha$-Isocinchonin mit Schwefelsäure vom specifischen Gewichte 1.125 auf $140-150^{\circ}$. Es ist in Äther leicht löslich, ist amorph, ebenso seine Salze, und wird erhaiten, wenn man die Mutterlauge des »Apocinchonins mit Äther extrahirt. Jungfleisch und Léger ${ }^{2}$ haben gefunden, dass es ein Gemenge

\footnotetext{
1 Liebig's Ann., 205, 333 und 276, 118.

2 Compt. rend., 114, 1192.
} 
von Basen ist, aus welchen sie und Andere $\alpha$ - und $\beta$-Isocinchonin (Cinchonigin und Cinchonilin) isoliren konnten. Hesse ${ }^{1}$ bezweifelt die Richtigkeit ihrer Angaben. Bei genauer Einhaltung der von Hesse gemachten Angaben habe ich aber bestätigt gefunden, dass aus dem "Diapocinchonin « das schwerlösliche saure Jodhydrat des $\alpha$-Isocinchonins und aus dessen Mutterlaugen das $\beta$-Isocinchonin als schwer lösliches, neutrales, salzsaures Salz zu isoliren ist. Es kann also nicht mehr bezweifelt werden, dass das Diapocinchonin ein Gemenge ist.

\section{Hydrochlorcinchonin.}

Hesse unterscheidet vier nach der Formel $\mathrm{C}_{19} \mathrm{H}_{23} \mathrm{ClN}_{2} \mathrm{O}$ und theils direct, theils indirect aus Cinchonin darstellbare Verbindungen, nämlich das Hydrochlorcinchonin, Hydrochlorapocinchonin, Hydrochlorapoisocinchonin und das Hydrochlor$\alpha$-Isocinchonin.

Die vierte Verbindung ist in Schmelzpunkt, Drehungsvermögen etc. so wesentlich verschieden von den erstgenannten drei Verbindungen, dass eine Verwechslung ausgeschlossen ist. Auf sie wird hier deshalb weiter keine Rücksicht genommen.

Die ersten drei Hydrochlorverbindungen haben mit einander aber eine so grosse Ähnlichkeit, dass ihre Verschiedenheit nicht in demselben Grade sicher ist, so insbesondere das Hydrochlorapoisocinchonin und das Hydrochlorcinchonin, für welche nur geringe Unterschiede im Schmelzpunkt und Löslichkeit angegeben werden, während Hydrochlorcinchonin und Hydrochlorapocinchonin nach Hesse physikalisch zwar in fast allen Stücken gleich, aber dadurch scharf unterschieden sind, dass das erstere, mit alkoholischer Kalilauge gekocht, ausschliesslich $\alpha$-Isocinchonin, das letztere dagegen ausschliesslich $\beta$-Isocinchonin gibt.

Hydrochlorcinchonin entsteht nach Hesse aus Cinchonin mit rauchender Salzsäure bei $85^{\circ}$, Hydrochlorapocinchonin bei $140^{\circ}$, das Hydrochlorapoisocinchonin dann, wenn $\alpha$-oder $\beta$-Isocinchonin mit bei $0^{\circ}$ gesättigter Salzsäure auf $140-150^{\circ}$ erhitzt 
wird. Die Identität oder Verschiedenheit der drei Verbindungen hat deshalb theoretisches Interesse. $v$. Kone ${ }^{1}$ hat vor einigen Jahren schon mitgetheilt, dass Hydrochlorcinchonin und -apocinchonin identisch sind; dieses ist aber von Hesse energisch bestritten worden. Es hat sich gezeigt, dass Konek's Angaben ganz richtig sind, und dass die Identität sich auch auf das Hydrochlorapoisocinchonin erstreckt.

Hydrochlorapoisocinchonin wurde genau nach Hesse's Angabe aus $\alpha$-Isocinchonin dargestellt. $9 \cdot 5 \mathrm{~g}$ Base gaben $2 \cdot 44 \mathrm{~g}$ des Dichlorhydrates. Die Mutterlauge dieser Krystallisation schied, mit $\mathrm{CH}_{3}$ zerlegt, neben $\alpha$-Isocinchonin $4 \cdot 3 \mathrm{~g}$ einer in Äther nicht löslichen Base ab, die, in einem Gemische von gleichen Raumtheilen concentrirter Salzsäure und Wasser gelöst, noch $1.8 g$ eines Dichlorhydrates von gleichem Ansehen gab, wie von dem erst erhaltenen.

Die beiden Salzfractionen sind im Aussehen vom Dichlorhydrate des Hydrochlorcinchonin, wie es nach der Vorschrift von Hesse entsteht, nicht $z u$ unterscheiden, lösen sich wie dieses in 5 Theilen kochenden Wassers und scheiden, mit $\mathrm{NH}_{3}$ zerlegt, eine Base ab, welche. aus Alkohol umkrystallisirt, ebenso wie das Hydrochlorcinchonin bei $213^{\circ}$ schmilzt. Auch die Löslichkeit in kochendem Alkohol ist bei allen drei Basen dieselbe, nähmlich ungefähr 1:100. Hesse hat angegeben, dass das freie Hydrochlorapoisocinchonin bei $203^{\circ}$, das Hydrochlorcinchonin bei $212^{\circ}$ schmelzen und ersteres in Alkohol ziemlich leicht löslich sein soll, andere Unterschiede aber nicht mitgetheilt. Wie es kam, dass von Hess e's Base bei $15^{\circ} 3$ Theile in 100 absoluten Alkohol in Lösung gingen, wie aus den Angaben über das Drehungsvermögen der Base hervorgeht, während meine genau nach seinen Angaben dargestellte in der Kochhitze erst in der 100 fachen Menge sich löste, kann ich nicht beurtheilen, und da Hesse selber angibt, dass Hydrochlorapoisocinchonin und Hydrochlorcinchonin, mit Kalilauge zerlegt, beide $\alpha$.Isocinchonin geben und nach meinen Beobachtungen die physikalischen Verhältnisse bei beiden Basen gleich sind, ist die Identität beider Verbindungen sichergestellt.

1 Monatshefte $1895,330$. 
Das Hydrochlorcinchonin ist aber auch mit dem Hydrochlorapocinchonin identisch. Die frappante Übereinstimmung beider Verbindungen in der meisten Richtung hat schon Hesse hervorgehoben, als Unterschied aber, wie schon erwähnt, angegeben, dass ersteres mit Kali zerlegt $\alpha$-Isocinchonin, letzteres $\beta$-Isocinchonin gibt.

Zur Untersuchung kam Hydrochlorapocinchonin, nach Hesse durch Erhitzen von Cinchonin mit hochconcentrirter Salzsäure auf $140^{\circ}$ dargestellt, und Hydrochlorcinchonin, nach Königs durch Einwirkung von Salzsäure bei gewöhnlicher Temperatur, die in Eis-Kochsalzmischung gesättigt war. Die Salze beider Basen wurden durch Umkrystallisiren aus Wasser gereinigt, sie waren in Wasser gleich löslich; die aus ihnen durch Ammoniak abgeschiedenen freien Basen zeigten den Schmelzpunkt 213 und auch in Löslichkeit und Aussehen vollständige Übereinstimmung.

Sie wurden in ganz derselben Weise mit alkoholischer Kalilauge gekocht. Auf je $5 \mathrm{~g}$ kamen $100 \mathrm{~cm}^{3}$ Alkohol und $40 \mathrm{~cm}^{3}$ einer $2 \cdot 8$ normalen alkoholischen Lauge; es wurde am Rückflusskühler, aber unter zeitweiliger Concentration gekocht. Das Ungelöste wurde in der Hälfte der Kochdauer filtrirt, durch Wasser vom Chlorkalium befreit und dann wieder zugefügt. Nach etwa vierstündigem Kochen war das Volumen $40 \mathrm{~cm}^{3}$; es wurde das gleiche Volum Wasser zugefügt, die abfiltrirte Lösung mit Äther ausgeschüttelt, dann durch Eindampfen vom Alkohol befreit und noch zweimal mit Äther extrahirt.

Die ätherischen Lösungen wurden vollständig abdestillirt, mit N-Salzsäure genau neutralisirt, das verbrauchte Volumen Salzsäure nochmals zugefügt, durch Abdampfen concentrirt und feingepulvertes Jodkalium in mässigem Überschuss eingeworfen. Das in hellgelben Krystallen ausgefallene saure Jodhydrat wurde abgesaugt und aus heissem Wasser umkrystallisirt. Sodann die beiden Filtrate mit $\ddot{t z k a l i}$ alkalisch gemacht, die freien Basen wieder mit Äther extrahirt, der Rückstand der Ätherlösung mit N-Salzsäure neutralisirt und eingedampft. Die auf Zusatz von Alkali mit ausgefallenen ätheruniöslichen Basen wurden den in verdünntem Alkohol unlöslichen zuggerechnet. 
Aus je $5 g$ Hydrochlorapo-, respective Hydrochlorcinchonin resultirten:

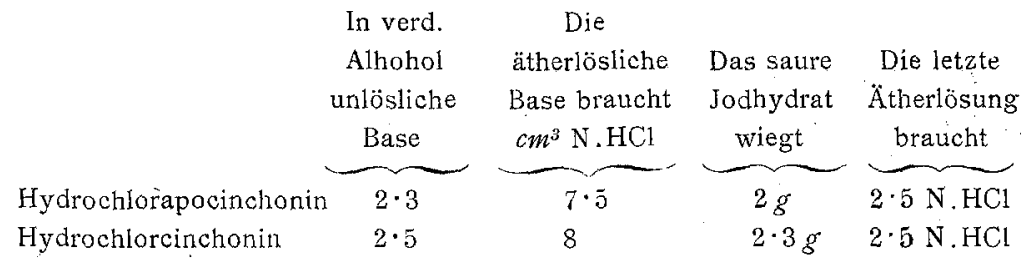

Die Jodhydrate waren in Farbe, Form und Löslichkeit in heissem Wasser $(1: 4 \cdot 7)$ identisch, und die aus ihnen abgeschiedenen Basen krystallisirten aus trockenem Äther in derben Krystallen vom Schmelzpunkte 126-127, so dass in beiden Fällen $\alpha$-Isocinchonin vorlag.

Die letzten ätherischen Extracte nach dem Neutralisiren mit Salzsäure eingedampft, blieben auch nach tagelangem Stehen amorph, enthalten daher $\beta$-Isocinchonin in nennenswerthen Mengen nicht, da dieses als neutrales Chlorhydrat sehr gut krystallisirt. Um jeden Irrthum auszuschliessen, habe ich den eben beschriebenen Versuch wiederholt und hiezu auch das nöthige Hydrochlorcinchonin und Hydrochlorapocinchonin noch einmal neu dargestellt, bin aber qualitativ zu ganz denselben Resultaten gelangt.

Beide Hydrochlorverbindungen zeigen daher den von Hess e angegebenen einzigen Unterschied nicht, und sind sie deshalb untereinander und, wie weiter oben schon gezeigt worden ist, auch mit dem Hydrochlorapoisocinchonin identisch. Selbstverständlich empfiehlt es sich, ron nun ab den kürzesten. Namen, d. i. Hydrochlorcinchonin für die Verbindung zu: gebrauchen. 